\title{
Matroid matching with Dilworth truncation
}

\author{
Márton Makai ${ }^{12 \dagger}$ \\ ${ }^{1}$ Dept. of Operations Research, Eötvös University, Pázmány P. s. 1/C, Budapest, Hungary H-1117. The author is a \\ member of the Egerváry Research Group (EGRES). e-mail: marci@cs . elte. hu. \\ ${ }^{2}$ Communication Networks Laboratory, Pázmány Péter sétány 1/A, Budapest, Hungary, H-1117.
}

Let $H=(V, E)$ be a hypergraph and let $k \geq 1$ and $l \geq 0$ be fixed integers. Let $\mathcal{M}$ be the matroid with ground-set $E$ s.t. a set $F \subseteq E$ is independent if and only if each $X \subseteq V$ with $k|X|-l \geq 0$ spans at most $k|X|-l$ hyperedges of $F$. We prove that if $H$ is dense enough, then $\mathcal{M}$ satisfies the double circuit property, thus the min-max formula of Dress and Lovász on the maximum matroid matching holds for $\mathcal{M}$. Our result implies the Berge-Tutte formula on the maximum matching of graphs ( $k=1, l=0)$, generalizes Lovász' graphic matroid (cycle matroid) matching formula to hypergraphs $(k=l=1)$ and gives a min-max formula for the maximum matroid matching in the 2-dimensional rigidity matroid $(k=2, l=3)$.

Keywords: matroid matching, Dilworth truncation, double circuit property

\section{Introduction}

The notion of matroid matching is known to be an involved class of combinatorial optimization problems concerning parity. One of its numerous equivalent definitions is as follows. Let $\mathcal{M}$ be a matroid with ground-set $E$, with rank-function $r_{\mathcal{M}}$, with span-function sp $\operatorname{M}_{\mathcal{M}}$ and let $A \subseteq\left(\begin{array}{c}E \\ 2\end{array}\right)$ be a set of (not necessarily disjoint) pairs of $E$. For short, if $F \subseteq E$ and $M \subseteq A$, then $r_{\mathcal{M}}(F \cup M)$ stands for $r_{\mathcal{M}}(F \cup \cup M)$ and $\operatorname{sp}(F \cup M)$ stands for $\operatorname{sp}(F \cup \cup M)$. A set of pairs $M \subseteq A$ is said to be a matroid matching of $A$ w.r.t. $\mathcal{M}$ if $r_{\mathcal{M}}(M)=2|M|$. The matroid matching problem is to compute a matroid matching of maximum size, the size of which is denoted by $\nu_{\mathcal{M}}(A)$.

Jensen and Korte (1982) and Lovász (1981) proved that the matroid matching problem cannot be solved in its full generality. On the other hand, the increasing number of its solvable special subclasses shows its particular importance.

Starting from the very special matching problem of graphs and the matroid intersection problem, good characterization of the maximum matroid matching Lovász (1980b) and also a polynomial algorithm Lovász (1981) was obtained by Lovász for represented linear matroids. This gives a method to compute the maximum matroid matching in the graphic matroid (cycle matroid), the minimum number of vertices of a graph to pin to obtain a 2-dimensional rigid graph, and the maximum genus of a graph. By a construction of Schrijver (2003), also Mader's maximum number of vertex-disjoint $\mathcal{A}$-paths can be computed

\footnotetext{
†Supported by OTKA grant T 037547 and TS 049788, by European MCRTN Adonet, Contract Grant No. 504438 and by the Egerváry Research Group of the Hungarian Academy of Sciences. Part of this work was done when the author was visiting Laboratoire Leibniz, Université Joseph Fourier, Grenoble.
}

1365-8050 (c) 2005 Discrete Mathematics and Theoretical Computer Science (DMTCS), Nancy, France 
by this. However, the min-max formula is given in a geometric language, it cannot be translated to a combinatorial one. This gap is partially filled by Lovász' structure theorem for 2-polymatroids Lovász (1980a) which enables us to derive combinatorial min-max formula for some of the above problems.

In the middle of the eighties, Dress and Lovász (1987) pointed out that the tractability of the known solvable cases is due to a more general common property of the above matroids. Up to this day, the double circuit property is the only general property that assures a method to compute the maximum matroid matching for every $A \subseteq\left(\begin{array}{c}E \\ 2\end{array}\right)$.

A set $U \subseteq E$ is said to be a double circuit of $\mathcal{M}$ if $r_{\mathcal{M}}(U)=|U|-2, r_{\mathcal{M}}(U-\{e\})=|U-\{e\}|-1=$ $|U|-2$ for every $e \in U$ and all the circuits of $U$ have positive rank. The double circuit $U$ has a simple structure, namely, it has a partition $U_{1} \dot{\cup} U_{2} \dot{\cup} \ldots \dot{U} U_{d}$ s.t. $C_{i}=U-U_{i}(i=1,2, \ldots, d)$ are all of its circuits. The double circuit is said to be non-trivial if $d \geq 3$. The crucial situation where most of the solution approaches to the matroid matching problem can get stuck is the existence of a non-trivial double circuit of a certain distinguished size. In this case, the possibility of reducing the problem to a "smaller" one has to be assured by the structural properties of the particular matroid we are dealing with. Lovász proved that in the case of full linear matroids, the modularity of lattice of flats (subspaces) is sufficient for this. However, there are cases where modularity does not hold but the maximum matching has a good characterization.

Dress and Lovász said that the matroid $\mathcal{M}$ has the double circuit property (DCP for short) if

$$
r_{\mathcal{M} / Z}\left(\bigcap_{1 \leq i \leq d} \operatorname{sp}_{\mathcal{M} / Z}\left(C_{i}\right)\right)>0
$$

holds for each non-trivial double circuit $U$ of each contraction $\mathcal{M} / Z$ of $\mathcal{M}$ (using the above notations). The following result is implicit in Lovász (1981, 1980a,b) and explicit in Dress and Lovász (1987).

Theorem 1 (Dress and Lovász (1987)) If $\mathcal{M}$ has the DCP and $A \subseteq\left(\begin{array}{c}E \\ 2\end{array}\right)$, then

$$
\nu_{\mathcal{M}}(A)=\min r_{\mathcal{M}}(Z)+\sum_{j=1}^{t}\left\lfloor\frac{r_{\mathcal{M} / Z}\left(A_{j}\right)}{2}\right\rfloor,
$$

where the minimum is taken for all flats $Z \subseteq E$ and for all partitions $A_{1}, A_{2}, \ldots, A_{t}$ of $A$.

The min-max formula has the same form as in Lovász (1980b) for linear matroids, but in the case of Dress and Lovász it is stated in a more general setting.

If the matroid $\mathcal{M}$ with ground-set $E$ is embedded into a matroid $\mathcal{M}^{\prime}$ with larger ground-set $E^{\prime} \supseteq E$ and $A \subseteq\left(\begin{array}{c}E \\ 2\end{array}\right)$, then $\nu_{\mathcal{M}}(A)=\nu_{\mathcal{M}^{\prime}}(A)$. Thus, the natural question which arises is to explore the class of matroids which have the DCP, and matroids with embedding into a larger matroid having the DCP. Dress and Lovász proved that full linear, full algebraic, full transversal, and full graphic matroids have the DCP, for definitions see Dress and Lovász (1987). In other words, every linear, algebraic, transversal, and graphic matroid has an embedding into a matroid which has the DCP.

Based on lattice theoretic concepts, Björner and Lovász (1987) introduced the class of pseudomodular matroids and they have shown that the above mentioned matroids are pseudomodular. Later, Hochstättler and Kern (1989) proved that pseudomodular matroids have the DCP.

Although the class of linear matroids, pseudomodular matroids, and matroids having the DCP are rather general, it can be difficult to prove that a particular matroid has an embedding into a matroid from one of these classes. If only linearity is known, then the matroid is embedded into the full linear space and combinatorial min-max formula cannot be expected. Moreover, some of the most important combinatorially 
defined matroids are linear but not known to be deterministically representable, which would be a requirement for computational results. Pseudomodular matroids form a special class of matroids having the DCP but searching for a pseudomodular embedding is not an easy task. Thus it remains a great challenge to explore combinatorially suggested tractable classes which give a more unified view of the solvable cases.

The rank-functions of matroids which are the most interesting from the application point of view are defined by Dilworth truncation of modular functions or functions defined by graphs or hypergraphs. Our main goal is to take a step in the way of better comprehension of the matching problem of such matroids. This is carried out by considering the matroid matching in the following class of purely combinatorially defined matroids. This may be a class where Dilworth truncation arise in the most simple way, but even this gives a more unified view of some solved cases and also contains previously unsolved problems.

Let $k \geq 1$ and $l \geq 0$ be fixed integers and let $H=(V, E)$ be a finite hypergraph. Let us define $b: 2^{V} \rightarrow \mathbb{Z}$ by $b(X)=k|X|-l$ if $k|X|-l \geq 0$ and 0 otherwise. For $X \subseteq V$ and $F \subseteq E$ let $F[X]=\{e \in F: e \subseteq X\}$. Finally, let $\mathcal{M}$ be the matroid with ground-set $E$ s.t. $F \subseteq E$ is independent in $\mathcal{M}$ if and only if $|F[X]| \leq b(X)$ for each $X \subseteq V$. We may suppose that each hyperedge is of size bigger than $\frac{l}{k}$ since the smaller hyperedges are loops. The hyperedges of size two will be called simply edges. For better comprehension, we note that the rank-function of $\mathcal{M}$ is expressed as

$$
r_{\mathcal{M}}(F)=\min \left\{|Y|+\sum_{X \in \mathcal{X}} b(X): Y \subseteq F, \mathcal{X} \subseteq\left(\begin{array}{c}
V \\
>\frac{l}{k}
\end{array}\right), F \subseteq Y \cup \bigcup_{X \in \mathcal{X}} E[X]\right\}
$$

The particular interest of the matroid matching of this matroid is due to the following more special problem. Let $H^{\prime}=\left(V, E^{\prime}\right)$ be a hypergraph, and we ask for the largest set $F^{\prime} \subseteq E^{\prime}$ s.t. $\left|F^{\prime}[X]\right| \leq \frac{k|X|-l}{2}$ for every $X \subseteq V, k|X|-l \geq 0$. Notice that, if $H^{\prime}$ is a graph, $k=1, l=0$, then this is exactly the matching problem of the graph $H^{\prime}$. For graphs and $k=l=2$, we get back the maximum forest problem. If $H^{\prime}$ is a 3-regular hypergraph, $k=l=1$ and $F^{\prime} \subseteq E^{\prime}$, then $\left|F^{\prime}[X]\right| \leq \frac{|X|-1}{2}$ holds for every $\emptyset \neq X \subseteq V$ if and only if the components of $F^{\prime}$ are triangle cacti. Hence, in 3-regular hypergraphs and $k=l=1$ we have the maximum forest problem. Jackson and Jordán proved that if $H^{\prime}$ is a graph and $k=5, l=7$, then the arising edge-sets are independent in the 3 -dimensional rigidity matroid. The importance of this result is that the rank-function of the 3-dimensional rigidity matroid is not known.

The above defined matroids can be shown to be linear, but for getting computational results from the application of Lovász' linear matroid matching theorem, the matroid has to be represented. However, if $H$ contains only edges, $k=2$ and $l=3$, then $\mathcal{M}$ is the 2-dimensional rigidity matroid (Laman (1970)) which is not known to be deterministically representable. If we are looking for a combinatorial min-max relation, then we cannot recline upon the linear embedding.

Thus, the matroid will be embedded into a relatively small, combinatorially defined matroid which has the DCP. This embedding is obtained by adding further hyperedges to $H$. As this operation does not affect $\nu_{\mathcal{M}}(A)$, we may assume for simplicity that the new hyperedges are already in $H$. The main goal of adding new hyperedges is to reach the desired structure of flats which have the matroids having DCP. The following property put a precise criterion for this.

Property 2 If $X \subseteq V$, then $r_{\mathcal{M}}(E[X])=b(X)$.

Our main theorems are the following.

Theorem 3 If Property 2 holds, then the matroid $\mathcal{M}$ has the DCP. 
Theorem 4 If Property 2 holds, $A \subseteq\left(\begin{array}{c}E \\ 2\end{array}\right)$, then for each contraction $\mathcal{N}$ of $\mathcal{M}$,

$$
\nu_{\mathcal{N}}(A)=\min r_{\mathcal{N}}(Z)+\sum_{j=1}^{t}\left\lfloor\frac{r_{\mathcal{N} / Z}\left(A_{j}\right)}{2}\right\rfloor,
$$

where the minimum is taken for all flats $Z \subseteq E$ of $\mathcal{N}$ and for all partitions $A_{1}, A_{2}, \ldots, A_{t}$ of $A$.

Theorem 4 immediately follows from Theorem 3 and Theorem 1.

As Property 2 is in the hypothesis of Theorem 4 we should give a short study of some criteria which imply Property 2. First, it is easy to see that if each set $X \subseteq V$ of size bigger than $\frac{l}{k}$ is in $E$ with multiplicity $k|X|-l$, then Property 2 holds. A weaker condition also assures Property 2, as it is described in the following theorem.

Theorem 5 Let $l=c k+d$ where $c, d$ are integers, $0 \leq c$ and $0 \leq d<k$. Suppose that $E$ contains all the subsets of $V$ of size $c+1$ with multiplicity $k-d$. Suppose moreover that if $\frac{c k}{c+1}<d$, then $E$ contains all the subsets of $V$ of size $c+2$ with multiplicity $c d+d-c k$. Then Property 2 holds.

For some applications, let $\varrho_{\mathcal{M}}(A)=\min \left\{|B|: B \subseteq A, r_{\mathcal{M}}(B)=r_{\mathcal{M}}(A)\right\}$. For any matroid $\mathcal{M}$, the computation of $\varrho_{\mathcal{M}}(A)$ is equivalent to computing $\nu_{\mathcal{M}}(A)$. This is formalized more specifically as follows.

Theorem 6 (Lovász (1981)) For any matroid $\mathcal{M}$ with ground-set $E$ and $A \subseteq\left(\begin{array}{c}E \\ 2\end{array}\right)$,

$$
\nu_{\mathcal{M}}(A)+\varrho_{\mathcal{M}}(A)=r_{\mathcal{M}}(A) .
$$

\section{Applications}

\subsection{Berge-Tutte formula and transversal matroid matching}

If $k=1$ and $l=0$, then Theorem 4 implies the Berge-Tutte formula Berge (1958). In the Introduction we sketched a construction which contains the matching problem of graphs. The following is a more simple construction. Let $E$ be the set of singletons of $V$. It can be seen immediately that Property 2 holds. The set of pairs for the matroid matching is $A=\left\{\{\{u\},\{v\}\}: u v \in E^{\prime}\right\}$. It is easy to see that $M^{\prime}$ is a matching of $G$ if and only if $M=\left\{\{\{u\},\{v\}\}: u v \in M^{\prime}\right\}$ is a matroid matching of $A$ w.r.t. $\mathcal{M}$.

Let $Z \subseteq E$ and $A_{1}, A_{2}, \ldots, A_{t}$ give equality in the min-max relation stated in Theorem 4 so that $\operatorname{sp}_{\mathcal{M}}(Z)$ is minimal, and subject to this, $t$ is as small as possible. Then, it can be proved that $Z$ will be a barrier in the Berge-Tutte formula and $\left\{u, v:\{\{u\},\{v\}\} \in A_{j}\right\}-Z, j=1,2, \ldots, t$ will be the set of vertex-sets of odd components and one of them is the union of the vertex-sets of even components.

One of the usual interpretations of transversal matroids is that we have a hypergraph $H=(V, E)$ and $F \subseteq E$ is independent if and only if $|F[X]| \leq|X|$ holds for every $X \subseteq V$. Similarly to the case of Berge-Tutte formula, if each singleton hyperedge is in $E$ with multiplicity one, then Property 2 holds, and Theorem 4 gives a good characterization for the maximum matching in transversal matroids.

We also have to note that the transversal matroid matching can be solved in an easier way. Tong et al. (1984) showed that even the weighted case can be reduced to the weighted matching problem of graphs. 


\subsection{Hypergraphic matroid and rigidity matroid}

We have mentioned in the Introduction that the maximum forest problem of 3-regular hypergaphs is also a special case. As in the case of the Berge-Tutte formula, this problem fits into our set-up in two different ways. We switch to that one which contains also the more general graphic matroid matching problem.

Let $1 \leq k \leq l \leq 2 k-1$. According to this choice, $c=1$ and $d=l-k$. To satisfy the requirements of Theorem $5, E$ has to contain $2 k-l$ parallel edges on each pair of vertices. If $d>\frac{c k}{c+1}=\frac{k}{2}$ or equivalently $l=k+d>\frac{3 k}{2}$, then we have to put $c d+d-c k=2 d-k=2 l-3 k$ parallel hyperedges of size three to each triple of vertices. Next we consider the applications of this case.

If $k=l=1$, then $\mathcal{M}$ is the hypergraphic matroid with ground-set $E$. As $l \leq \frac{3 k}{2}$, then Property 2 holds if $\left(\begin{array}{c}V \\ 2\end{array}\right) \subseteq E$. If $E$ contains only edges, then Theorem 4 specializes to Lovász' theorem on the maximum graphic matroid matching Lovász (1980a).

Theorem 7 (Lovász (1980a)) $A \subseteq\left(\begin{array}{c}V \\ 2\end{array}\right)$ (i.e. $\mathcal{M}$ is the cycle matroid of the graph $\left(V,\left(\begin{array}{c}V \\ 2\end{array}\right)\right)$ ). Then

$$
\nu_{\mathcal{M}}(A)=\min |V|-|\mathcal{P}|+\sum_{j=1}^{t}\left\lfloor\frac{r_{\mathcal{N}}\left(A_{j}\right)}{2}\right\rfloor,
$$

where the minimum is taken for all partitions $\mathcal{P}=\left\{P_{1}, P_{2}, \ldots, P_{q}\right\}$ of $V$ and for all partitions $A_{1}, A_{2}, \ldots, A_{t}$ of $A$ and $\mathcal{N}$ is the cycle matroid of the graph obtained from $\left(V, \cup A_{j}\right)$ by contracting the members of $\mathcal{P}$.

If $\bigcup A$ contains also hyperedges of size bigger than two, then Theorem 4 cannot be rewritten in such a special form. In this case, the contraction cannot be described by a partition of $V$. To see this, let $e_{0}, e_{1}, \ldots, e_{m}, m \geq 3$ be pairwise vertex-disjoint hyperedges of size three and $A=\left\{\left\{e_{0}, e_{i}\right\}: 1 \leq\right.$ $i \leq m\}$. Then the only possibility of obtaining equality in the min-max formula is $Z=\left\{e_{0}\right\}$ and $A_{j}=\left\{\left\{e_{0}, e_{j}\right\}\right\}, 1 \leq j \leq t=m$.

If $k=2$ and $l=3$, then $F \subseteq E$ is independent in $\mathcal{M}$ if and only if $|F[X]| \leq 2|X|-3$ for every $X \in\left(\begin{array}{c}V \\ \geq 2\end{array}\right)$. Notice that, if $\bigcup A$ contains only edges, then this is the "smallest" case when Theorem 4 gives a new result. Just as above, Property 2 is satisfied if $\left(\begin{array}{l}V \\ 2\end{array}\right) \subseteq E$. If $E$ contains only edges, then it is known that the bases of $\mathcal{M}$ are exactly the 2-dimensional minimally rigid graphs on $V$ (Laman (1970)). Let $G=\left(V, E^{\prime}\right)$ be a 2-dimensional rigid graph and let $A$ be a set of (not necessarily disjoint) pairs from $E^{\prime}$. Then the maximum number of edge-pairs from $A$ which are contained in a minimally rigid subgraph of $G$ is $\nu_{\mathcal{M}}(A)$, which can be computed by Theorem 4. If $G=\left(V, E^{\prime}\right)$ is not a rigid graph but $\left(V, E^{\prime} \cup \cup A\right)$ is rigid, where $A \subseteq\left(\begin{array}{c}V \\ 2\end{array}\right)$, then $\varrho_{\mathcal{M} / E^{\prime}}(A)$ is the minimum cardinality of $B \subseteq A$ s.t. $\left(V, E^{\prime} \cup \cup B\right)$ is rigid. This problem can be solved by Theorem 4 and Theorem 6.

For larger $k$ and $l$, Property 2 does not follows from $\left(\begin{array}{l}V \\ 2\end{array}\right) \subseteq E$. Say, if $k=3$ and $l=5$, then a hyperedge of size three have to be put to each triple of vertices.

\subsection{A connectivity augmentation problem}

The problems discussed here were proposed by Zsolt Fekete. Let $G=\left(V, E^{\prime}\right)$ be an undirected graph, let $1 \leq k \leq l \leq 2 k-1$ and $\mathcal{M}$ be as above. Let moreover an other edge-set $E^{\prime \prime}$ on $V$ and a set of packets $A \subseteq 2^{E^{\prime \prime}}$ be given. We ask for the minimum cardinality set $B \subseteq A$ s.t. the rank of $\left(V, E^{\prime} \cup \cup B\right)$ is $k|V|-l$ in $\mathcal{M}$. Clearly, if $A$ is composed by singletons, then this is a minimum cardinality spanning subset problem in a matroid. 
Frank observed (personal communication) that if each packet is composed by $p$ parallel edges, $p=$ $k=l$ and $k$ is part of the input, then then the problem is NP-hard. The graph on 2 vertices obtained from $G$ after contracting $|V|-2$ pairs of vertices consecutively contains $k$ edge-disjoint spanning trees if and only if $G$ has a cut of size at least $k$. Hence, this reduces the maximum cut problem to our problem.

If $p=2$ and $k \geq 1, l \geq 0$ are arbitrary integers, then we just have to compute $\varrho_{\mathcal{M} / E^{\prime}}(A)$. This contains the problem is of adding a minimum number of capacity 2 edges (from a prescribed set) to $G$ so that the resulting graph has $k$ edge disjoint spanning trees $(k=l)$. By Theorem 4 and Theorem 6 , a combinatorial characterization is achieved.

\section{Acknowledgements}

The author wishes to thank Zsolt Fekete, András Frank, Zoltán Király and Jácint Szabó for useful discussions on the topic.

\section{References}

C. Berge. Sur le couplage maximum d'un graphe. C. R. Acad. Sci. Paris, 247:258-259, 1958.

A. Björner and L. Lovász. Pseudomodular lattices and continuous matroids. Acta Sci. Math. (Szeged), 51 (3-4):295-308, 1987.

A. Dress and L. Lovász. On some combinatorial properties of algebraic matroids. Combinatorica, 7(1): 39-48, 1987.

W. Hochstättler and W. Kern. Matroid matching in pseudomodular lattices. Combinatorica, 9(2):145-152, 1989.

B. Jackson and T. Jordán. The $d$-dimensional rigidity matroid of sparse graphs. J. Combin. Theory Ser. B. to appear.

P. M. Jensen and B. Korte. Complexity of matroid property algorithms. SIAM J. Comput., 11(1):184-190, 1982.

G. Laman. On graphs and rigidity of plane skeletal structures. J. Engrg. Math., 4:331-340, 1970.

L. Lovász. Matroid matching and some applications. J. Combin. Theory Ser. B, 28(2):208-236, 1980a.

L. Lovász. Selecting independent lines from a family of lines in a space. Acta Sci. Math. (Szeged), 42 (1-2):121-131, 1980b.

L. Lovász. The matroid matching problem. In Algebraic methods in graph theory, Vol. I, II (Szeged, 1978), volume 25 of Colloq. Math. Soc. János Bolyai, pages 495-517. North-Holland, Amsterdam, 1981.

A. Schrijver. Combinatorial optimization. Polyhedra and efficiency. Vol. C, volume 24 of Algorithms and Combinatorics. Springer-Verlag, Berlin, 2003. Disjoint paths, hypergraphs, Chapters 70-83.

P. Tong, E. L. Lawler, and V. V. Vazirani. Solving the weighted parity problem for gammoids by reduction to graphic matching. In Progress in combinatorial optimization (Waterloo, Ont., 1982), pages 363-374. Academic Press, Toronto, ON, 1984. 